\title{
O CONSENSO DO CONSENSO ESTRATÉGICO: UM ESTUDO SOBRE O ESTADO DA ARTE DO TEMA
}

\section{RESUMO}

Este trabalho objetiva apresentar o estado da arte sobre consenso estratégico, ressaltando as abordagens utilizadas, a natureza do método de pesquisa utilizado, os resultados encontrados e a agenda futura para estudos do tema. Analisando as últimas quatro décadas de publicações, concluiu-se que relacionar o consenso estratégico somente com a performance e com os níveis estratégicos pode ser entendido como algo limitado, o que sugere a necessidade de trazer para o campo da investigação novos aspectos e contextos como inovação, processo de geração de novas ideias, a ocorrência do consenso além do nível de Top Management Team (TMT), entre outros. Além disso, também conclui-se que a abordagem predominante do consenso estratégico é por processo, bem como a metodologia utilizada é baseada em técnicas quantitativas. Como sugestão de estudos futuros, o presente estudo indica a investigação de situações em que o consenso estratégico não é positivo.

Palavras-chave: Consenso; Estratégia; Tomada de Decisão.

THE CONSENSUS OF STRATEGIC CONSENSUS: A STUDY OF THE STATE OF THE ART ABOUT THE THEME

\begin{abstract}
This paper aims to present the state of the art regarding the strategic consensus, emphasizing the approaches and the nature of the research methods used, the results obtained and the future agenda for this theme studies. Analyzing beyond the last four decades of publications, it was understood that relating the strategic consensus only with the performance and strategic levels can be seen as something limited, suggesting the need to bring to the researching field new aspects and backgrounds as innovation, the methods for generating new ideas, the occurrence beyond the Top Management Team level (TMT), among others. Moreover, concludes that the predominant approach the strategic consensus is a process and the methodology used is based on quantitative techniques. As a suggestion for future studies, this study indicates the investigation of situations in which the strategic consensus is not positive.
\end{abstract}

Keywords: Consensus; Strategy; Decision-Making. 


\section{EL CONSENSO DEL CONSENSO ESTRATEGICO: UN ESTUDIO RESPECTO AL ESTADO DEL ARTE}

\section{DEL TEMA}

\section{RESUMEN}

Este trabajo objetiva presentar el estado del arte respecto al consenso estratégico, señalando los abordajes, las naturalezas del método de investigaciónes, los resultados encontrados y la agenda futura para los estudios del tema. Analizando las últimas cuatro décadas de publicaciones, se concluye que relacionar el consenso estratégico solo con el rendimiento y con los niveles estratégicos puede entenderse como algo limitado, lo que sugiere la necesidad de aportar al campo de la investigación nuevos aspectos y contextos como la innovación, el proceso de generación de nuevas ideas, la ocurrencia del consenso más allá del nivel de Top Management Team (TMT), y otros. Además, se concluye igualmente que el abordaje predominante del consenso estratégico es procesual, así como la metodología utilizada está basada en técnicas cuantitativas. Como sugerencia para futuras investigaciones, el presente estudio indica la investigación de situaciones en que el consenso estratégico no es positivo.

Palabras clave: Consenso; Estrategia; Tomada de Decisión.

Marcelo Curth ${ }^{1}$ Renata Araujo Bernardon² Guilherme Trez ${ }^{3}$

\footnotetext{
${ }^{1}$ Doutor em Administração pela Universidade do Vale do Rio dos Sinos - UNISINOS. Professor da Universidade FEEVALE. Brasil. E-mail: m.curth1979@gmail.com

2 Doutoranda em Administração pela Universidade do Vale do Rio dos Sinos - UNISINOS. Professora da Pontifícia Universidade Católica do Rio Grande do Sul - PUC/RS. Brasil. E-mail: renatabernardon@hotmail.com

${ }^{3}$ Doutor em Administração pela Universidade Federal do Rio Grande do Sul - UFRGS. Professor da Universidade do Vale do Rio dos Sinos - UNISINOS. Brasil. E-mail: gtrez@unisinos.br
} 


\section{INTRODUÇÃO}

O conceito de consenso vem ganhando considerável popularidade nos trabalhos relacionados ao contexto estratégico (Ho, Wu \& Wuc, 2014; Murphy \& O'Brien, 2014), pois observa-se que nas últimas quatro décadas um crescente número de publicações tem tratado sobre o tema do consenso estratégico.

Considerando os estudos, ressalta-se as diferentes abordagens utilizadas pelos pesquisadores a citar os estudos de Stagner (1969), o qual entendia o consenso como concordância, os de McMahon e Ivancevich (1976), que trataram do consenso gerencial e a influência do mesmo no desempenho dos liderados, os de Quinn (1982), em que o autor busca o entendimento sobre a importância do consenso nas ações gerenciais estratégicas, os de Dess e Origer (1987), que apresentaram um importante framework tratando o relacionamento do consenso na formulação da estratégia e na performance, e os de Wooldridge e Floyd (1989), que buscaram explicar a falta de relacionamentos consistentes dos trabalhos anteriores sobre o consenso do Top Management Team [TMT] e a performance organizacional.

Além disso, alguns estudos encontraram resultados conflitantes no que tange consenso e estratégia, sendo os casos de Gorman, Rosa e Faseruk (2005), que encontraram como resultado que o consenso para empréstimo estava relacionado com o relacionamento e conhecimento de um seleto grupo de analistas de crédito e os solicitantes. Bem como os estudos de Farjaudon e Morales (2013), os quais ressaltam como contadores moldam o que é consensual gerando regras para a atuação da profissão e Ambrosini e Bowman (2003), que ressaltaram a pouca importância do consenso em causas como ambiguidade ou falta de interesse por parte dos participantes, entre outros.

Porém, uma grande quantidade de artigos converge para o mesmo sentido em relação aos seus resultados de pesquisa, os quais ressaltam a importância do consenso para a estratégia, sendo os casos de Floyd e Wooldridge (1992), Phelps, Harris e Johnson (1996), Harris, Dopson e Fitzpatrick (2009), entre outros.

$\mathrm{O}$ interesse contínuo no tema consenso e estratégia, bem como as evidências conflitantes sobre o tema sugerem a necessidade de uma síntese empírica dos resultados e abordagens anteriores. Assim, o presente trabalho objetiva apresentar o estado da arte do tema consenso estratégico, ressaltando as abordagens utilizadas, os temas tratados, a natureza de pesquisa, os resultados encontrados e a agenda futura para estudos do tema.

As contribuições deste trabalho possibilitam o entendimento de que o tema do consenso estratégico ainda é um campo com potencial de pesquisa, pois ao longo de mais de quarenta anos, as pesquisas deram continuidade às anteriores que focavam na relação do consenso estratégico e a performance. Os estudos mais recentes ampliaram a abordagem do consenso estratégico, trazendo para o campo da investigação novos aspectos e contextos, tais como inovação, processo de geração de novas ideias, a ocorrência além do nível de Top Management Team [TMT], entre outros.

Neste período, foram apresentados modelos, frameworks e matrizes que buscam explicar o consenso estratégico, considerando as abordagens utilizadas, a natureza do método de pesquisa utilizada, os resultados encontrados e a agenda futura para estudos do tema. Porém, estas estruturas ainda não estão consolidadas, inclusive seus respectivos autores indicam a aplicação das mesmas em outras realidades e em escala maior para que assim se possa realizar a generalização dos resultados.

Mesmo que os artigos observados apresentem uma diversidade de naturezas metodológicas, bem como uma grande variedade de amostras e objetos de estudo, é possível afirmar que existe uma tendência para a o enfoque do consenso estratégico como processo, ou seja, quando o consenso estratégico não tem um fim em si mesmo e leva /influencia a uma outra etapa da prática estratégica. Em relação à abordagem do consenso estratégico como processo, também pode ser percebida a intensa adoção de métodos quantitativos. Porém, quando o tema em questão é abordado como resultado, ou seja, quando algum elemento leva ao consenso estratégico, a metodologia escolhida pelos autores de forma predominante, foram as técnicas qualitativas.

O artigo contará com três sessões, além desta introdução. O método é apresentado na segunda sessão, contendo aspectos sobre como e onde foi realizada a procura pelos materiais sobre o tema. A terceira sessão apresenta fundamentação teórica, ressaltando os temas consenso e estratégia. A quarta sessão traz a discussão, a conclusão e as considerações finais seguidas das referências utilizadas neste artigo.

\section{MÉTODO}

Como recurso metodológico, este trabalho utilizou os estudos do estado da arte, pois tais estudos possibilitam um maior conhecimento da produção acadêmica sobre o tema. Os estudos do tipo estado da arte permitem ao pesquisador sistematizar um determinado campo do conhecimento ou tema, reconhecer os principais resultados, identificar temáticas e abordagens dominantes/emergentes, bem como as lacunas de novas pesquisas, utilizando como base um determinado espaço de tempo (Haddad, 2000).

O período compreendido para a busca ocorreu entre os anos de 1969 e 2014. O período de início da 
busca justifica-se pela publicação seminal de Stagner (1969), sendo tal estudo tido como inovador nas práticas de tomada de decisão (Kellermanns, et al., 2011). No entanto, por conta da maior ocorrência e aderência dos estudos relacionados ao consenso estratégico surgirem a partir de 1980, o presente estudo explorou as publicações referentes ao tema a partir de tal década. Para identificar todos os estudos disponíveis sobre consenso estratégico, foram realizadas pesquisas com palavras-chave em bases de dados. As bases utilizadas foram Ebsco, Elsevier, Proquest e Emerald. $\mathrm{Na}$ busca foram utilizadas as palavras-chave consensus e strategy. Como resultado da busca nas bases de dados, foram encontrados artigos em journals $\mathrm{e}$ periódicos de diferentes áreas como gestão (serviços, pública e informação), saúde, psicologia, tecnologia e contabilidade, totalizando o número de 539 artigos.

Para a depuração dos artigos, foram adotados dois critérios, sendo eles a aderência à área de estratégia organizacional e a abordagem referente ao tema em estudo. Ambos os critérios foram considerados após a leitura da introdução/delimitação do tema dos trabalhos. Após a análise dos artigos, foram identificados 40 estudos que atenderam aos dois critérios. Desses artigos, 33\% apresentaram suas publicações concentradas nas seguintes revistas: Journal of Business Research, Journal of Operations Management, Journal of Business Venturing, Academy of Management Executive, Strategic Management Journal e Journal of Business Strategy.

Após realizada a seleção dos 40 artigos, os mesmos foram analisados sistematicamente buscando identificar o objetivo, a metodologia adotada, as conclusões e as sugestões de estudos futuros. Feita esta identificação e também com o maior entendimento a que se propunha cada um dos artigos, os autores do presente estudo puderam classificar os artigos nas duas grandes abordagens que o consenso estratégico pode ser compreendido: sendo estas o consenso como resultado do processo estratégico, ou o consenso como processo de sua construção em si (Dess \& Origer, 1987).

\section{CONSENSO E SEU ESTADO DA ARTE}

Um dos conceitos aceitáveis e que traduzem o consenso é de que trata-se da concordância das partes envolvidas na tomada de decisão (Dess \& Origer, 1987). Os autores ressaltam ainda que o consenso pode ocorrer somente após o processo de discussão entre pontos positivos e negativos das questões envolvidas, e quando todos (não uma maioria) dos envolvidos estão em concordância.

Além do conceito do consenso, é de grande importância o conhecimento sobre as dimensões e as questões investigadas na natureza do consenso apresentadas entre o Top Management Team [TMT], pois muitos dos trabalhos publicados no campo da estratégia estão relacionados com tais pontos. Segundo Wooldridge e Floyd (1989), o foco dos trabalhos sobre consenso trata as dimensões em termos de grau ou nível (forte; fraco), escopo (quem são os participantes) e de conteúdo (o que os tomadores concordam).

No que tange a natureza das investigações, Dess e Origer (1987) destacam que a produção sobre o tema consenso, até o período da investigação dos artigos desses autores, tinha como foco o consenso como resultado do processo de tomada de decisão, ou o consenso como processo de construção do mesmo. Entende-se o consenso como resultado quando algum elemento leva ao consenso (poder, confiança e valores, por exemplo). Já o consenso como processo é caracterizado quando o consenso leva a uma outra etapa (tomada de decisão ou desenvolvimento estratégico, por exemplo).

Dess (1987), destaca que os estudos anteriores relacionados ao consenso estratégico foram realizados por Laurence e Lorsch (1967), Stagner (1969), Holder (1976), Grinyer e Norburn (1977) e DeWoot, Heyvaert e Martou (1977). Nestes estudos, o objetivo consistia em investigar aspectos relacionados à performance dos gestores e da organização como um todo. $\mathrm{O}$ consenso surgiu como resultado de tais estudos e as expressões utilizadas eram altos níveis de integração (Laurence \& Lorsch, 1967), coesão (Stagner,1969), tomada de decisão (Holder, 1976) e concordância (Grinyer \& Norburn, 1977; DeWoot, Heyvaert \& Martou, 1977). Os mesmos não traziam uma linha de construção de um conceito sobre o consenso ou como ele era formado de forma explícita.

No entanto, os estudos realizados na sequência dos acima citados trazem diferentes temas, metodologias e resultados relevantes para o entendimento do consenso e a sua relação com as diferentes variáveis do contexto estratégico organizacional. Com a observação destes estudos e seus respectivos temas, percebeu-se que a análise sob o ponto de vista de décadas e enfoque da abordagem são relevantes para a compreensão do estado da arte do tema em estudo.

Neste sentido, a tabela 1 foi organizada para possibilitar a verificação da distribuição quantitativa por décadas dos 40 artigos observados, bem como a concentração dos estudos em relação à abordagem. Observando a tabela 1, percebe-se que os estudos referentes ao consenso estratégico estão sendo intensificados nas últimas décadas. Cabe ressaltar que a década de 2010 é composta por apenas 4 anos, diferente das demais que são compostas por 10 cada. Assim, tem-se a expectativa que ao término desta última década tenha-se maior quantidade de publicações relacionadas ao tema. 
Tabela 1 - Abordagens e principais temas do consenso estratégico

\begin{tabular}{|c|c|c|c|c|}
\hline Década & $\begin{array}{c}\mathrm{N}^{\circ} \text { de } \\
\text { artigos }\end{array}$ & Abordagem & $\begin{array}{c}\text { Principais } \\
\text { Temas }\end{array}$ & Principais Resultados \\
\hline \multirow[t]{2}{*}{1980} & 4 & Processo & $\begin{array}{l}\text { - Relação do consenso entre } \\
\text { TMT e: (i) a performance } \\
\text { econômica e (ii) os objetivos } \\
\text { e métodos competitivos. } \\
\text { - Processo de tomada de } \\
\text { decisão baseadas em } \\
\text { consenso versus decisão por } \\
\text { voto. } \\
\text { - Consequências das } \\
\text { abordagens de tomada de } \\
\text { decisão em grupo ou por } \\
\text { voto. } \\
\text { - Framework integrativo } \\
\text { sobre a relação do consenso } \\
\text { da formulação da estratégia e } \\
\text { a performance. }\end{array}$ & $\begin{array}{l}\text { - A falta de consenso sobre os meios é mais } \\
\text { problemática do que o desacordo sobre os fins } \\
\text { (Bourgeois, 1980). } \\
\text { - Importância de processos para a melhoria na } \\
\text { qualidade de informações para tomada de } \\
\text { decisões (Quinn, 1982). } \\
\text { - A busca pelo consenso durante o processo de } \\
\text { decisão pode induzir ao comprometimento } \\
\text { individual e coletivo, reduzindo as divergências } \\
\text { em situações de competição (Tjosvold \& Field, } \\
\text { 1983). } \\
\text { - Proposições sobre a relação do consenso } \\
\text { organizacional, métodos competitivos e o } \\
\text { ambiente, além do nível de desempenho, } \\
\text { características de mercado e a integração da } \\
\text { estrutura (Dess \& Origer, 1987). }\end{array}$ \\
\hline & 2 & Resultado & $\begin{array}{l}\text { - Entendimento do consenso } \\
\text { pelos gestores estratégicos. } \\
\text { - Elementos influenciadores } \\
\text { do consenso estratégico. }\end{array}$ & $\begin{array}{l}\text { - O consenso em relação aos objetivos ou } \\
\text { métodos são positivos para a performance, não } \\
\text { sendo necessário ter consenso sobre ambos (Dess, } \\
\text { 1987). } \\
\text { - Consenso em estratégia deve ser visto como } \\
\text { algo de grande amplitude e não deve ser } \\
\text { generalizado para se chegar ao resultado } \\
\text { (Wooldridge \& Floyd, 1989). }\end{array}$ \\
\hline \multirow[t]{2}{*}{1990} & 4 & Processo & $\begin{array}{l}\text { - O consenso como } \\
\text { abordagem para diagnosticar } \\
\text { problemas de formulação } \\
\text { estratégica. } \\
\text { - Temas pertinentes para a } \\
\text { busca do consenso. } \\
\text { - A influência do consenso } \\
\text { estratégico na prática } \\
\text { estratégica. } \\
\text { - Influência da diversidade } \\
\text { demográfica e o processo de } \\
\text { grupo no consenso } \\
\text { estratégico no TMT. }\end{array}$ & $\begin{array}{l}\text { - Entendimento e compromisso estratégico } \\
\text { compartilhados são a base estratégias e ações } \\
\text { coerentes e eficazes (Floyd \& Wooldridge, 1992). } \\
\text { - } \quad \text { Geração de uma guideline para grupos que } \\
\text { queiram buscar consenso em relação aos recursos } \\
\text { (Marino, 1996). } \\
\text { - Crescente utilização do coletivo para o } \\
\text { consenso na estratégia de comunicação (Phelps, } \\
\text { Harris \& Johnson, 1996). } \\
\text { - A diversidade demográfica influencia } \\
\text { negativamente o consenso (Knight, et al., 1999). }\end{array}$ \\
\hline & 2 & Resultado & $\begin{array}{l}\text { - Consenso sobre elementos } \\
\text { para vantagem competitiva } \\
\text { no franchising. } \\
\text { - Consenso sobre os } \\
\text { resultados a partir dos } \\
\text { conteúdos. }\end{array}$ & $\begin{array}{l}\text { - Relação entre franqueador e franqueado } \\
\text { apresentam relação positiva com a performance } \\
\text { (Baucus, Baucus \& Human, 1996). } \\
\text { - Surgimento de níveis de consenso, } \\
\text { considerando assuntos fundamentais (diferenciais } \\
\text { competitivos, infraestrutura e estrutura de } \\
\text { investimentos) (Boyer \& McDermott, 1999). }\end{array}$ \\
\hline
\end{tabular}


Tabela 1 - (continuação)

\begin{tabular}{|c|c|c|c|c|}
\hline Década & $\begin{array}{c}\mathrm{N}^{\mathbf{0}} \text { de } \\
\text { artigos }\end{array}$ & Abordagem & $\begin{array}{c}\text { Principais } \\
\text { Temas }\end{array}$ & Principais Resultados \\
\hline \multirow[t]{2}{*}{2000} & 8 & Processo & $\begin{array}{l}\text { - A influência do consenso } \\
\text { estratégico na prática } \\
\text { estratégica. } \\
\text { - Consenso como apoio } \\
\text { para a viabilidade de } \\
\text { comunicação. } \\
\text { - Construção do consenso } \\
\text { em empresas familiares e não } \\
\text { familiares. } \\
\text { - Consenso nas avaliações } \\
\text { de risco financeiro. } \\
\text { - Relação entre os } \\
\text { geradores de consenso e a } \\
\text { criação de ideias. } \\
\text { - Comparação de processo } \\
\text { de busca do consenso entre o } \\
\text { modelo japonês e holandês. } \\
\text { • Processo de decisão em } \\
\text { diferentes conselhos de } \\
\text { administração. } \\
\text { - Direcionamentos } \\
\text { estratégicos e o papel no } \\
\text { planejamento. }\end{array}$ & $\begin{array}{l}\text { - A formulação do consenso ocorre pelo } \\
\text { incremento do escopo do consenso e não pela sua } \\
\text { construção (Markóczy, 2001). } \\
\text { • Empresas que são capazes de evocar fortes } \\
\text { ligações de comunicação são beneficiadas pelo } \\
\text { consenso estratégico (Rapert, Velliquette } \\
\text { \&Garretson, 2002). } \\
\text { - Nos grupos familiares é mais simples de } \\
\text { atingir o consenso do que grupos não familiares } \\
\text { (Ensley \& Pearson, 2004). } \\
\text { - O consenso e a existência de uma cultura de } \\
\text { empréstimo sensível às necessidades das } \\
\text { empresas baseadas no conhecimento de } \\
\text { especialistas de crédito (2005). } \\
\text { - Geradores de consenso podem sofrer do } \\
\text { excesso de pensamento coletivo, que pode ser } \\
\text { suscetível para não gerar boas ideias } \\
\text { (Vandenbosch \& Saatcioglu, 2006). } \\
\text { - O consenso é interpretado de forma diferente } \\
\text { entre os grupos pesquisados, pois os gestores } \\
\text { japoneses são mais ordenados e sequenciais que } \\
\text { os holandeses (Nooderhaven, Benders \& Keizer, } \\
\text { 2007). } \\
\text { - A informação trazida por um novo diretor tem } \\
\text { maior impacto quando comparado ao impacto da } \\
\text { nova diretoria sobre a negociação quando o } \\
\text { conselho é grande e não diversificado (Baranchuk } \\
\text { \& Dybvig, 2008). } \\
\text { - Com a elaboração deliberada de múltiplas, } \\
\text { ambíguas e ambiciosas estratégias, os gestores } \\
\text { são capazes de efetuar a mudança organizacional } \\
\text { e não pela implementação da estratégia literal, } \\
\text { mas usando essas estratégias como metáforas para } \\
\text { potencializar o consenso e legitimidade dos } \\
\text { principais grupos interessados (Harris, Dopson \& } \\
\text { Fitzpatrick, 2009). }\end{array}$ \\
\hline & 4 & Resultado & $\begin{array}{l}\text { - Formulação do consenso } \\
\text { para tomada de decisão na } \\
\text { produção. } \\
\text { - Nível de consenso sobre } \\
\text { elementos relacionados à } \\
\text { estratégia. } \\
\text { - Elementos influenciadores } \\
\text { do consenso. } \\
\text { - Importância do consenso } \\
\text { sobre a estratégia utilizando a } \\
\text { teoria da agência. }\end{array}$ & $\begin{array}{l}\text { - Não há relação direta entre alinhamento das } \\
\text { prioridades de produção e a performance da } \\
\text { unidade (Joshi, Kathuria \& Porth, 2003). } \\
\text { • Não generalização do consenso na existência } \\
\text { de uma lógica estratégica pouco conhecida ou } \\
\text { cenários de incerteza (Ambrosini \& Bowman, } \\
\text { 2003). } \\
\text { - A diversidade funcional possui efeito positivo } \\
\text { na inovação quando há um contexto de consenso } \\
\text { estratégico na equipe de gestão (Camelo-ordaz, } \\
\text { Hernándes-lara \& Valle-cabrera, 2005). } \\
\text { • A congruência de metas baseadas na aceitação } \\
\text { da estratégia e incentivos de reforço podem } \\
\text { resultar em um sistema de controle menos } \\
\text { oneroso e mais eficiente (Bouillon, et al., 2006). }\end{array}$ \\
\hline
\end{tabular}

(Continuação) 
Tabela 1 (continuação)

\begin{tabular}{|c|c|c|c|c|}
\hline Década & $\begin{array}{c}\mathrm{N}^{\mathbf{0}} \text { de } \\
\text { artigos }\end{array}$ & Abordagem & $\begin{array}{c}\text { Principais } \\
\text { Temas }\end{array}$ & Principais Resultados \\
\hline \multirow[t]{2}{*}{2010} & 11 & Processo & $\begin{array}{l}\text { - Nível de educação e } \\
\text { diversidade como } \\
\text { influenciador da inovação. } \\
\text { - Consenso como elemento } \\
\text { da prática estratégica. } \\
\text { - Impacto do consenso } \\
\text { sobre as atribuições e atitudes } \\
\text { de marca. } \\
\text { - Processo de consenso na } \\
\text { tomada de decisão em } \\
\text { ambientes difusos. } \\
\text { - Processo de mudança de } \\
\text { comunicação para o } \\
\text { consenso. } \\
\text { - Avaliação crítica do uso } \\
\text { de técnicas Delphi em } \\
\text { pesquisas qualitativas do } \\
\text { consenso. } \\
\text { - Processo de } \\
\text { desenvolvimento do } \\
\text { consenso. } \\
\text { - Papel da contabilidade na } \\
\text { formação do consenso. } \\
\text { - Processo de consenso } \\
\text { como função legitimadora. } \\
\text { - A decisão estratégica e o } \\
\text { valor potencial na tomada de } \\
\text { decisão. } \\
\text { - A construção do consenso } \\
\text { no sucesso das estratégias. }\end{array}$ & $\begin{array}{l}\text { • Nível de educação do TMT influência } \\
\text { inovação e consenso (Camelo-ordaz, Fernández- } \\
\text { alles \& Hernández-lara, 2010). } \\
\text { - A identificação social é mediadora das práticas } \\
\text { de alta performance e da satisfação no trabalho } \\
\text { (Young et al., 2010). } \\
\text { - A alegação do consenso é uma estratégia de } \\
\text { publicidade eficaz, mas seu impacto varia com a } \\
\text { informação e a motivação do processamento da } \\
\text { audiência (Freling \& Dacin, 2010). } \\
\text { - Proposta de modelo para captar a imprecisão e } \\
\text { incerteza dos pensamentos dos envolvidos } \\
\text { (Khorshid, 2010). } \\
\text { - Influência das dualidades: auto interesse das } \\
\text { partes x bem coletivo, emergente consenso das } \\
\text { partes x líder impulsionador, inclusão das partes x } \\
\text { exclusão (Stoltzfus, Stohl \& Seibold, 2011). } \\
\text { - A utilização das técnicas é utilizado em locais } \\
\text { onde o consenso é pouco provável de ser criado } \\
\text { (Paraskevas \& Saunders, 2011). } \\
\text { - Condições para beneficiar o desenvolvimento } \\
\text { de consenso no desenvolvimento de inovações } \\
\text { arquitetônicas interindustriais (Jasper, Prencipe \& } \\
\text { Van Den Edge, 2012). } \\
\text { - A contabilidade consolida as assimetrias nas } \\
\text { posições de poder, moldando o consenso } \\
\text { (Farjaudon \& Morales, 2013). } \\
\text { - O consenso pode ser uma forma particular de } \\
\text { governar (Byrne \& Shrestha, 2014). } \\
\text { - As partes envolvidas no processo constroi o } \\
\text { consenso sobre a decisão de investimentos de } \\
\text { projetos (Murphy \& O’Brien, 2014). } \\
\text { - A construção do consenso, participação } \\
\text { efetiva, institucionalização, liderança e } \\
\text { capacitação são determinantes no sucesso da } \\
\text { estratégia (Rasoolimanesh, Jaafar \& } \\
\text { Badarulzaman, 2014). }\end{array}$ \\
\hline & 5 & Resultado & $\begin{array}{l}\text { - Elementos influenciadores } \\
\text { do consenso. } \\
\text { - Formulação do consenso. } \\
\text { - Consenso como elemento } \\
\text { de composição da estratégia. } \\
\text { - Efeito do consenso no } \\
\text { desempenho organizacional. } \\
\text { - Influência do consenso na } \\
\text { implementação da estratégia. }\end{array}$ & $\begin{array}{l}\text { - Executivos sêniors e gerentes de produção } \\
\text { discordam sobre a importância das prioridades de } \\
\text { produção (Kathuria, et al., 2010). } \\
\text { - Novas diretrizes no consenso organizacional } \\
\text { (Prusty, Mohapatra \& Mukherjee, 2010). } \\
\text { - O consenso leva as organizações a serem } \\
\text { tolerantes ao risco (Pretorius \& Maritz, 2010). } \\
\text { - O consenso estratégico e o desempenho são } \\
\text { moderados pelo dinamismo ambiental } \\
\text { (Kellermanns, et al., 2011). } \\
\text { - O consenso em organizações orientadas para o } \\
\text { cliente está associado com o desempenho de } \\
\text { funcionários de linha de frente (Ho, Wu \& Wuc, } \\
\text { 2014). }\end{array}$ \\
\hline Total & 40 & & & \\
\hline
\end{tabular}

Fonte: Dados da pesquisa 
Outro fato curioso de ser observado é a maior intensidade de estudos que abordam o consenso estratégico como um processo quando comparada com a intensidade dos estudos que abordam o tema em questão como resultado.

Assim, a seguir, serão descritos cada um dos períodos em maior detalhe tanto no que diz respeito aos temas tratados, principais conclusões e indicações de estudos futuros e a relação destes com suas respectivas metodologias (qualitativa, quantitativa ou estudo teórico) e abordagens (por processo ou resultado).

\subsection{O consenso na década de 80}

A década de 80 inaugurou os estudos relacionados ao consenso e a estratégia de forma mais densa e constante em termos de publicações. Neste período, a preocupação da pesquisa acadêmica abordou fortemente a relação do consenso e a performance organizacional (Bourgeois, 1980; Dess, 1987; Dess \& Origer, 1987; Wooldridge \& Floyd, 1989). A influência do consenso no gerenciamento do processo de formulação da estratégia global (Quinn, 1982) e a preocupação em relação a eficiência da adoção das técnicas de tomada de decisão baseadas em consenso comparadas com a decisão por voto (Tjosvold \& Field, 1983) também foram abordados.

O processo de decisão baseado no consenso parece ser mais moroso e menos eficiente por tomar uma quantidade de tempo maior quando comparado com a decisão por voto. Porém, a decisão por consenso induz a um comprometimento maior, tanto em nível individual quanto em grupo (Tjosvold \& Field, 1983). O que reforça esta indicação da adoção de técnicas de processo de decisão baseados no consenso é a positiva relação da melhora da performance organizacional quando se tem consenso em relação aos objetivos estratégicos (Dess, 1987), e principalmente em relação aos meios pelos quais serão atingidos (Bourgeois, 1980).

No final da década de 80 , percebe-se que o consenso em estratégia deve ser entendido em sentido mais amplo do que se estava imaginando. Relacionar o consenso somente com a performance e com os níveis estratégicos pode ser uma abordagem limitada (Wooldridge \& Floyd, 1989). Dessa forma, é preciso observar o consenso organizacional em relação aos métodos para o alcance do consenso, características de mercado de atuação e integração de estruturas empresarias que propiciem seu alcance (Dess \& Origer, 1987). Assim, durante este período as pesquisas realizadas sugerem direcionar os estudos para a busca da generalização dos resultados, realizando comparações entre indústrias para determinar se as associações entre consenso e performance organizacional são específicos dos setores estudados ou são aplicáveis à uma variedade de ambientes competitivos (Dess, 1987; Wooldridge \& Floyd, 1989). Também é sugerido a abordagem do tema em relação ao conteúdo estratégico, processo estratégico e performance (Dess \& Origer, 1987).

Os trabalhos que abordaram o consenso por processo somaram 4 artigos nesta década, com destaque para as pesquisas quantitativas ( 3 trabalhos) e uma das pesquisas com abordagem teórica. A pesquisa de abordagem teórica teve sua publicação no final da década e utilizou os artigos anteriores, tendo como proposta central a relação entre consenso e a performance (Dess \& Origer, 1987). Já as pesquisas quantitativas abordaram a relação do consenso por processo com performance (Bourgeois, 1980; Dess, 1987) e sobre o consenso e elementos, como a qualidade do compromisso compreensão do problema, tempo para a tomada de decisão e atitudes em relação ao grupo (Tjosfold \& Field, 1983). Outro elemento importante das pesquisas da década é a não realização de estudos qualitativos sobre o consenso por processo.

No entanto, os trabalhos de consenso como resultado nesta década utilizaram o método qualitativo, sendo o caso de (Quinn, 1982). O trabalho foi o único da década a utilizar o método qualitativo, sugerindo a inclinação do método para a abordagem do consenso como resultado. Outro método utilizado na abordagem por resultado nesta década foi o teórico (Wooldridge \& Floyd, 1989). Coincidentemente os trabalhos abordaram temas semelhantes da relação do processo estratégico e do consenso por resultado.

A tabela 2 apresenta os periódicos com maior destaque, considerando o número de publicações no período. O periódico com maior destaque foi o Strategic Management Journal, trazendo 3 artigos, com destaque para o consenso por resultado. 
Tabela 2 - Periódicos com publicação

\begin{tabular}{lccc}
\multicolumn{1}{c}{ Journals } & Processo & Resultado & Total \\
\hline Academy of Management Journal & 1 & & 1 \\
OMEGA - The International Journal of Management & 1 & 2 & 1 \\
Strategic Management Journal & 1 & & 1 \\
The Academy of Management Review & 1 & $\mathbf{2}$ & $\mathbf{6}$
\end{tabular}

Fonte: Autores

Assim, a década destacada contabilizou 6 estudos sobre o tema consenso, com maior incidência dos artigos com método quantitativo, os quais foram realizados no consenso por processo. Nesse sentido, inicia-se o entendimento de que os estudos quantitativos poderiam estar vinculados ao consenso como processo. Além disso, destaca-se que os artigos teóricos foram realizados tanto no consenso por processo quanto por resultado, sugerindo abertura para estudos posteriores utilizando este método em ambas as abordagens do consenso.

\subsection{O consenso na década de 90}

Os estudos referentes ao consenso e a estratégia na década de 90 foram marcados pela busca da compreensão de quais são as condições necessários para que o consenso ocorra (Wooldridge \& Floyd, 1992), sobre o que é preciso buscar o consenso nas organizações (Marino, 1996; Baucus, Baucus \& Human, 1996), de quem é a atribuição na organização para fomentar o consenso (Phelps, Harris \& Johnson, 1996), o que acaba por influenciá-lo (Knight et al., 1999) e porque é importante as organizações buscarem o consenso estratégico (Boyer \& McDermott, 1999).

Os resultados encontrados nesta segunda década de estudos indicam que existem áreas estratégicas da organização em que há desacordo substancial entre os níveis da empresa (Boyer \& McDermott, 1999), o que acaba afetando o seu desempenho. Assim, é preciso que ocorra uma orientação para a busca do consenso em relação a quais são os recursos realmente competitivo da organização (Marino, 1996), que entendimentos e compromissos estratégicos compartilhados são a base para uma ação coerente e uma estratégia eficaz (Wooldridge \& Floyd, 1992) e que é de todos a responsabilidade pela busca do consenso (Phelps, Harris \& Johnson, 1996). Nesta década também foi identificado que a diversidade dos membros que compõem o TMT (Top Management Team) pode influenciar negativamente no alcance do consenso estratégico (Baucus, Baucus \& Human, 1996).

Nesta década, os autores indicam como necessidade de pesquisas explorar de forma mais densa a relação do consenso com o desenvolvimento e execução da estratégia organizacional (Wooldridge \& Floyd, 1992), bem como verificar se intervenções diretas destinadas a promover o trabalho em equipe e cooperação no âmbito dos TMT (Top Management Team), pois pode mitigar eventuais efeitos negativos da diversidade de membros que compõem os TMT, além de analisar a influência dos diferentes níveis de poder no âmbito interno do TMT no consenso estratégico (Knight et al., 1999). É importante ressaltar que a recomendação já realizada na década anterior, a qual ressaltava a necessidade de buscar a generalização dos resultados permanece (Phelps, Harris \& Johnson, 1996), deixando-os mais audaciosos ainda, uma vez que é sugerido não somente a adaptação de estudos realizados além de outras indústrias, mas também ampliar a abordagem do consenso para contextos de cooperativas e acordos de cooperação entre países (Baucus, Baucus \& Human, 1996). Pelas sugestões de estudos futuros de Boyer e McDermott (1999), percebe-se que a necessidade de esclarecer a relação entre a performance organizacional e o consenso permanece, porém de forma ampliada, uma vez que os autores indicam a análise das percepções de diferentes áreas funcionais dentro de uma mesma empresa.

Seguindo o número de publicações da década anterior, os artigos de consenso por processo desta década somaram 2 quantitativos e 2 qualitativos. Porém outros autores abordaram objetivos diferentes, tratando do consenso na tomada de decisão (Phelps, Haris \& Johnson, 1996), e a integração dos conceitos da alta hierarquia (Knight et al., 1999).

Nos artigos que abordaram o consenso como processo e utilizaram método qualitativo, verificou-se um aumento no número de publicações nesta década comparada a década anterior. Os artigos tiveram como objetivo mostrar o consenso estratégico como forma de diagnosticar problemas na formulação de estratégias (Floyd \& Wooldridge, 1992) e analisar a relação entre consenso e recursos da organização (Marino, 1996).

Com relação aos trabalhos da abordagem do consenso como resultado, a quantidade seguiu a década anterior (2). O método quantitativo foi utilizado pelos únicos trabalhos da abordagem nesta década. O trabalho teve como objetivo investigar como a 
diversidade demográfica e o processo de grupo influenciam o consenso estratégico no TMT (Baucus, Baucus \& Human, 1996) e do argumento teórico para se chagar ao consenso (Boyer \& McDermott, 1999).

Além das informações já apresentadas torna-se importante o entendimento de outros aspectos sobre o comportamento das publicações da época. Nesse sentido, as tabelas abaixo possibilitam identificar as características das pesquisas do período, trazendo os periódicos da época. No caso dos periódicos da Tabela 3, ressalta-se o aumento de publicações na Academy of Management Executive, com pesquisas abordando a abordagem de processo do consenso. Além disso, ressalta-se a presença de pesquisas tratando de resultado na Strategic Management Journal.

Tabela 3 - Periódicos com publicação

\begin{tabular}{lccc}
\multicolumn{1}{c}{ Journals } & Processo & Resultado & Total \\
\hline Academy of Management Executive & 2 & & 2 \\
Journal of Business Research & 1 & & 1 \\
Journal of Business Venturing & & 1 & 1 \\
Journal of Operations Management & & 1 & 1 \\
Strategic Management Journal & & 1 & 1 \\
\hline \multicolumn{1}{c}{ Total Geral } & $\mathbf{3}$ & $\mathbf{3}$ & $\mathbf{6}$
\end{tabular}

Fonte: Autores

Em suma, a produção desta década foi semelhante em números absolutos de trabalhos em relação a década anterior, porém com diferenças nos métodos utilizados, bem como nas propostas de análise. Além disso, destaca-se o aumento no número de trabalhos qualitativos no consenso por processo e a não publicação de trabalhos teóricos durante a década.

\subsection{O consenso na década de 2000}

Os estudos referentes ao consenso e a estratégia nesta década trataram, a exemplo do período anterior, sobre o que é preciso para buscar o consenso nas organizações (Markóczy, 2001), e de quem é a atribuição na organização em fomentar o consenso (Ambrosini \& Bowman, 2003). Porém, alguns estudos emergiram preenchendo as lacunas deixadas e ressaltas no período anterior, sendo os casos dos estudos com foco na relação do consenso estratégico entre gerentes gerais e gerentes de produção e o aumento da performance (Joshi, Kathuria \& Porth, 2003) e processos de decisão em conselhos de administração (Baranchuk \& Dybvig, 2008).

No entanto, também emergiram estudos de temáticas com as necessidades latentes da época, com destaque para os trabalhos sobre a importância da comunicação para o consenso intraorganizacional (Rapert, Velliquette \& Garretson, 2002), o estudo da influência do consenso estratégico na estratégia de inovação (Ordaz, Lara \& Cabrera, 2005), os geradores de consenso na criação de novas ideias (Vanderbosch \& Saatcioglu, 2006), o consenso em empresas familiares e não familiares (Ensley \& Pearson, 2004), consenso na avaliação de risco financeiro (Gorman, Rosa \& Faseruk, 2005) e comparando o consenso em diferentes modelos organizacionais (Noorderhaven, Benders \& Keizer, 2007).

Dentre os principais resultados encontrados nesta década, encontram-se os de Markóczy (2001) o qual ressalta que a formulação do consenso se dá quando existe um conjunto de interesses comuns que beneficiará o grupo. Outros autores encontraram resultados que ressaltam a importância da comunicação (Rapert, Velliquette \& Garretson, 2002), a ambiguidade e desconhecimento das estratégias (Ambrosini \& Bowman, 2003; Harris, Dopson \& Fizpatrick, 2009) e importância dos fatores culturais (Noorderhaven, Benders \& Keizer, 2007).

Alguns dos resultados encontrados estão relacionados com os ambientes, pois ressaltam os moderadores do consenso nas prioridades de fabricação (Joshi, Kathuria \& Porth, 2003). Um dos resultados que chamou a atenção fora os de Camelo-ordaz, Hernández-lara \& Valle-cabrera (2005) que perceberam que níveis elevados de consenso estratégico podem influenciar negativamente em processos que envolvam inovação.

Os autores desta década indicam como necessidade de pesquisa a análise de influência de outras variáveis moderadoras, como fundos funcionais de gerentes e cultura organizacional, bem como comparativos entre culturas gerenciais (Noorderhaven, Benders \& Keizer, 2007), ressaltando culturas emergentes como exemplo (Gorman, Rosa \& Faseruk, 2005). Além disso, também foi sugerido examinar os efeitos moderadores de diferenças nos níveis hierárquicos de respondentes nivelados sobre a relação de alinhamento estratégico e o desempenho (Joshi, Kathuria \& Porth, 2003). 
Nesta década é curioso observar que tanto a abordagem dos estudos quanto os métodos adotados foram mais homogêneos quando comparados com os estudos das duas décadas anteriores. Em relação a abordagem por processo ou resultado, obteve-se 8 estudos para as abordagens. Dos 8 estudos com abordagem do consenso estratégico como processo 5 foram qualitativos, 2 quantitativos e 1 teórico. Ambos os métodos buscaram explorar enfoques que ainda não haviam sido abordados anteriormente tais como a importância do consenso na comunicação entre os níveis gerenciais e a relação do consenso sobre as metas e a performance do sistema de gestão em si. Um exemplo disso foram os trabalhos qualitativos que buscou compreender de forma exploratória se durante a mudança estratégica ocorre a construção do consenso (Markóczy, 2001). Outros dois estudos qualitativos, exploraram elementos influenciadores do consenso (Vandenbosch \& Saatcioglu, 2006; Noorderhaven, Benders \& Keizer, 2007).

Os trabalhos que apresentaram abordagem do consenso estratégico como resultado somaram 4 artigos e todos apresentaram estudos quantitativos. Como resultados, os estudos trouxeram que não há relação direta entre alinhamento das prioridades de produção e a performance da unidade (Joshi, Kathuria \& Porth, 2003) a não generalização do consenso na existência de uma lógica estratégica pouco conhecida ou cenários de incerteza (Ambrosini \& Bowman, 2003), que a diversidade funcional possui efeito positivo na inovação quando há um contexto de consenso estratégico na equipe de gestão (Camelo-ordaz, Hernández-lara \& Valle-cabrera, 2005) e que a congruência de metas baseadas na aceitação da estratégia e incentivos de reforço podem resultar em um sistema de controle menos oneroso e mais eficiente (Bouillon, et al., 2006).

Ressalta-se as aplicações utilizando estatísticas mais complexas e com maior atenção às medidas e validades das escalas utilizadas (Bouillon, Ferrier, Stuebs Jr. \& West, 2006) e a grande quantidade de artigos da época que não realizaram sugestões para estudos futuros, apenas a replicação sem direcionamentos para as suas propostas. Além disso, a Tabela 4 apresenta o número de publicações relativos aos periódicos do período, destacando o aumento em quantidade dos periódicos e a manutenção dos que já foram mencionados nos períodos anteriores.

Tabela 4 - Periódicos com publicação

\begin{tabular}{|c|c|c|c|}
\hline Journals & Processo & Resultado & Total \\
\hline Entrepreneurship Theory and Practice & 1 & & 1 \\
\hline European Management Journal & & 1 & 1 \\
\hline Journal of Accounting and Public Policy & & 1 & 1 \\
\hline Journal of Business Research & 1 & & 1 \\
\hline Journal of Business Strategy & 1 & & 1 \\
\hline Journal of Business Venturing & 1 & & 1 \\
\hline Journal of Management Development & & 1 & 1 \\
\hline Journal of Management Studies & 1 & & 1 \\
\hline Journal of Operations Management & & 1 & 1 \\
\hline Oxford University Press & 1 & & 1 \\
\hline Public Administration and Development & 1 & & 1 \\
\hline Strategic Management Journal & 1 & & 1 \\
\hline Total geral & 8 & 4 & 12 \\
\hline
\end{tabular}

Fonte: Autores

Por fim, ainda destaca-se que na década de 2000 registra-se um incremento de $100 \%$ do número de publicações relacionadas ao consenso estratégico, totalizando 12 publicações, enquanto as duas décadas anteriores apresentaram o mesmo número de 6 publicações.

\subsection{Consenso na década de 2010}

O último período analisado contemplou os anos entre 2010 e 2014. Em especial, os anos de início e fim do período foram os que concentraram maior número de publicações sobre o tema consenso estratégico. Neste período, a exemplo do período anterior, a preocupação da pesquisa acadêmica sobre o consenso estratégico apresentou novidades nas abordagens propostas. No entanto, não faltaram os estudos sobre nível de consenso dos executivos sêniores e gerentes de produção na definição de prioridades de produção 
(Kathuria, et al., 2010), a relação do consenso e a performance organizacional (Stoltzfus, Stohl \& Seibold, 2011; Kellermanns, et al., 2011), e também sobre performance de liderados (Ho, Wu \& Wu, 2014).

No entanto, assim como a década anterior, também emergiram abordagens importantes como modelos de consenso flexíveis entre tomadores de decisão (Khorshid, 2010), consenso compondo a prática estratégica (Pretorius \& Maritz, 2011) e como técnicas que envolvem o consenso para detecção de crise (Paraskevas \& Saunders, 2011). Um grupo de estudos chamou a atenção por incluir abordagens que ressaltam diferentes stakeholders nas abordagens de estudos, sendo o caso do trabalho sobre o consenso no desenvolvimento de inovações arquitetônicas utilizando abordagem interindustriais (Jaspers, Prencipe \& Den Edge, 2012), do consenso para investimentos e projetos (Murphy e O’Brien, 2014) e do consenso no desenvolvimento estratégico de cidades (Rasoolimanesh, Jaafar \& Badarulzaman, 2014).

Outro estudo que chamou novamente a atenção, pois já havia sido destacado na década anterior (Camelo-ordaz, Hernández-lara \& Valle-cabrera, 2005), foi o realizado por Camelo-ordaz, Fernándezalles \& Hernández-lara (2010), que analisaram como o nível de educação e a diversidade do top Management Team (TMT), moderado pelo consenso estratégico, influencia a performance da inovação.

Os resultados encontrados no período sobre consenso e performance endossam os já encontrados nos períodos anteriores, ressaltando que o consenso estratégico está associado positivamente com o desempenho organizacional (Kellermanns, et al., 2011). Porém, também foram destacados resultados que, assim como estudos de anos anteriores, apontam o surgimento de variáveis importantes mediadoras entre o consenso estratégico e a performance, tais como fatores sociais (Young, et al., 2010) e o dinamismo ambiental e desempenho dos funcionários de linha de frente (Stoltzfus, Stohl \& Seibold, 2011; Ho, Wu \& $\mathrm{Wu}, 2014)$. Além disso, outros resultados apontam no sentido da importância do modelo de consenso flexível para ser utilizado em ambientes difusos (Khorshid, 2010) e da coordenação, integração e interesse entre os especialistas envolvidos no processo interindustrial, pois facilita a tomada de decisão e resolvem os problemas relacionados aos possíveis conflitos (Jaspers, Prencipe \& Den Edge, 2012; Murphy \& O'brien, 2014).

Durante este período, as pesquisas realizadas sugerem direcionar os estudos para a busca de novas abordagens como a análise da gama de paradoxos dos stakeholders associados com a mudança organizacional planejada (Stoltzfus, Stohl \& Seibold, 2011).Outras sugestões de estudos futuros apontam na direção de estudos de décadas anteriores sobre a necessidade de inclusão de variáveis moderadoras em relação ao consenso (Kellermanns, et al., 2011; Rasoolimanesh, Jaafar \& Badarulzaman, 2014). As solicitações relacionando consenso e performance não foram evidenciadas nesta década analisada. Vale ressaltar que tal abordagem vinha sendo sugerida e analisada nos últimos 40 anos. Outro ponto que merece destaque trata da falta de criatividade e/ou aprofundamento nas discussões de alguns dos trabalhos analisados com relação às sugestões de estudos futuros. A maioria dos estudos ressaltava apenas a utilização de natureza metodológica complementar e o aumento no tamanho da amostra.

No que diz respeito à abordagem e métodos desta década, é possível verificar que ocorreu uma maior concentração em relação à abordagem do consenso estratégico como processo com 11 estudos dos 16 totais desta década e do aumento de métodos quantitativos, totalizando 5 estudos. Porém, os estudos que abordaram o consenso como resultado, mesmo que em minoria, apresentaram mais estudos quantitativos, tendo a sua distribuição da seguinte maneira: 1 estudo qualitativo, 5 quantitativo. Os estudos qualitativos e quantitativos também buscaram compreender e identificar os elementos influenciadores do consenso estratégico, assim como já vem ocorrendo desde a década de 80.

Ainda em relação à abordagem do consenso estratégico como processo neste período, dos 5 estudos qualitativos presentes, 2 buscaram explorar um novo tema, o consenso como elemento da prática de gestão e o outro estudo deu sequência à exploração dos diversos elementos em que o consenso pode exercer influência. Neste estudo em específico realizado por Jaspers, Prencipe e Den Edge (2012), os autores exploraram a influência do consenso nos processos de inovação. Já os 4 estudos quantitativos que abordaram o consenso estratégico como prática, concentraram sua investigação na compreensão das diferentes situações em que o consenso possa influenciar, apresentando somente 2 estudos que buscaram quantificar o consenso como elemento da prática estratégica. Já o estudo teórico de Khorshid (2010), propôs um modelo para o desenvolvimento de consenso flexível entre os tomadores de decisão no âmbito de ambiente difuso. A Tabela 5 apresenta o resultado dos periódicos com publicações sobre a abordagem, considerando o período analisado. 
Tabela 5 - Periódicos com publicação

Journals

Accounting, Organizations and Society

Cities

Critical Perspectives on Accounting

Expert Systems with Applications

International Development Planning Review

International Journal of Contemporary Hospitality

International Journal of Manpower

International Journal of Operations \& Production

Journal of Business Research

Journal of Business Strategy

Journal of Consumer Psychology

Journal of Health Organization and Management

Journal of Organizational Change Management

Journal of Production and Innovation Management

Management Research Review

Technological Forecasting \& Social Change

Total geral

$\begin{array}{ccc}\text { Processo } & \text { Resultado } & \text { Total } \\ 1 & 1 & 1 \\ 1 & & 1 \\ 1 & & 1 \\ 1 & & 1 \\ 1 & & 1 \\ 1 & & 1 \\ & & 1 \\ & 1 & 1 \\ & 1 & 1 \\ 1 & 1 & 1 \\ 1 & & 1 \\ 1 & & 1 \\ 1 & & 1 \\ 1 & & 1 \\ & & 1 \\ \mathbf{1 1} & 1 & 1 \\ & \mathbf{5} & \mathbf{1 6}\end{array}$

Fonte: Autores

Destaca-se, assim como a década anterior, o aumento em termos de quantidade de periódicos que abordaram o tema sobre o consenso.

A análise ressalta o aumento das pesquisas por processos e a preferência por uma das abordagens dos periódicos que estavam presentes nas décadas anteriores, sendo os casos dos periódicos Journal of Business Research e Journal of Business Strategy. Com os resultados desta década é possível verificar uma provável tendência dos estudos relacionados ao consenso estratégico adotarem uma abordagem por processo e fazerem uso de métodos quantitativos.

\section{DISCUSSÃO DOS RESULTADOS}

Considerando o abordado nas seções anteriores, entende-se haver diferenças de ênfase nas preocupações dos pesquisadores considerando os períodos abordados. Na década de 1980, a preocupação dos pesquisadores centrou-se fortemente na relação do consenso e na performance organizacional. Porém, relacionar o consenso somente com a performance e com os níveis estratégicos pode ser entendido como algo limitado, sugerindo novas abordagens para períodos posteriores. Tal sugestão estendeu-se para os outros períodos, em especial nas décadas de 1990 e 2000. Já nos estudos da década de 1990, os estudos referentes ao consenso e a estratégia foram marcados pela busca da compreensão de quais são as condições necessários para que o consenso ocorra. Nesta década, os autores indicam como necessidade de pesquisas explorar de forma mais densa a relação do consenso com o desenvolvimento e execução da estratégia organizacional e as intervenções no trabalho em equipe e nos níveis de poder no âmbito dos TMT.

Na década entre 2000 e 2010, alguns estudos emergiram preenchendo as lacunas deixadas e ressaltas no período anterior. Também emergiram estudos de temáticas com as necessidades latentes da época como consenso na comunicação entre organizações, inovação, criação de novas ideias e ressaltam os ambientes turbulentos como ponto importante para a abordagem do consenso estratégico.

Entre 2011 e 2014, a exemplo do período anterior, a preocupação da pesquisa acadêmica sobre o consenso estratégico apresentou novidades nas abordagens propostas, não faltando os artigos sobre performance que haviam diminuído a quantidade de publicações nas décadas anteriores. Algumas abordagens surgiram tratando da influência dos diferentes stakeholders, da legitimação de profissões na produção do consenso e da legitimação de compromissos e funções políticas.

Com a análise dos artigos realizada, foi possível identificar tendências em relação às abordagens, à natureza dos métodos de pesquisa adotados, os principais temas e a agenda futura de estudos relacionados ao consenso estratégico. 
Assim, com o objetivo de evidenciar e apresentar as informações citadas e comentadas ao longo do capítulo anterior apresenta-se a Tabela 1.

A Tabela 1 apresenta a visão evolutiva da abordagem (processo ou resultado) em relação à natureza do método adotado (qualitativa, quantitativa ou estudo teórico). Nesta tabela é possível verificar que ao longo das 4 décadas estudadas a abordagem do consenso estratégico por processo sempre foi predominante em relação a abordagem por resultado. $\mathrm{Na}$ década de 2000, foi quando ocorreu um relativo equilíbrio em relação as abordagens, porém na década de 2010 a tendência de concentração dos estudos sobre o consenso estratégico como processo volta a ocorrer e é confirmada.

No que diz respeito ao método utilizado para investigar o tema em estudo, percebe-se a adoção intensiva de métodos quantitativos. Dos 40 estudos observados, 22 (55\%) foram quantitativos e ao longo das décadas sempre foram predominantes. É interessante observar a presença constante de estudos teóricos ao longo das décadas, o que pode indicar que de tempo em tempo os pesquisadores da área percebem a necessidade de realizar um apanhado dos principais achados e indicar as possíveis lacunas e tendências existentes no campo da pesquisa do consenso estratégico.

Tabela 6 - Evolução da abordagem e da metodologia do consenso estratégico.

\begin{tabular}{|c|c|c|c|c|c|}
\hline Década & Abordagem & Qualitativa & Quantitativa & Estudo Teórico & Total Abordagem \\
\hline \multirow{2}{*}{1980} & Processo & 1 & 2 & 1 & 4 \\
\hline & Resultado & & 1 & 1 & 2 \\
\hline \multirow{2}{*}{1990} & Processo & 2 & 2 & & 4 \\
\hline & Resultado & & 2 & & 2 \\
\hline \multirow{2}{*}{2000} & Processo & 5 & 2 & 1 & 8 \\
\hline & Resultado & & 4 & & 4 \\
\hline \multirow{2}{*}{2010} & Processo & 5 & 5 & & 11 \\
\hline & Resultado & 1 & 4 & 1 & 5 \\
\hline Total Método & & 14 & 22 & 4 & 40 \\
\hline
\end{tabular}

Fonte: Dados da pesquisa.

É importante ressaltar que dos 40 estudos observados, 27 abordaram o consenso estratégico como processo e destes, 11 foram estudos quantitativos, 13qualitativos e 3 teóricos. Seguindo a mesma lógica, relata-se que 13 foi o número global de estudos que observaram o consenso estratégico como resultado, e destes 1 foi qualitativo, 11 quantitativos e 1 teórico. Assim, é possível mais uma vez reforçar a tendência da predominância de estudos que compreendem o consenso estratégico como processo e a adoção por métodos qualitativos. A novidade diz respeito a abordagem do tema em estudo por resultado, que contrário à abordagem por processo, é possível perceber uma maior predominância metodológica de estudos quantitativos. Isso pode ser explicado observando o tema recorrente de pesquisas com esta característica (consenso estratégico como resultado) que é o estudo dos elementos que influenciam o consenso estratégico. Este tema foi o enfoque de 10 dos 13 estudos realizados, e esteve presente ao longo das 4 décadas observadas.

Voltando a observar a Tabela 6, é possível verificar que quando o consenso estratégico é abordado como processo, o principal tema enfatizado é a influência do consenso estratégico na prática estratégica. Este tema apresentou 12 estudos ao todo, sendo 10 quantitativos e 2 qualitativos, o que mais uma vez reforça a tendência que vem sendo descrita inúmeras vezes.

\section{CONCLUSÕES}

Os estudos realizados sobre o consenso estratégico até meados do início da década de 1980 demonstraram que este tema pode estar relacionado ao processo de tomada de decisão, de formulação estratégica, de negociação entre outros. No entanto, estudos posteriores trouxeram diferentes abordagens, metodologias e resultados importantes para o entendimento do consenso e a sua relação com as diferentes variáveis para a tomada de decisão no contexto estratégico organizacional.

Ainda observando o tema do consenso estratégico, outro ponto relevante a ser observado diz respeito ao comportamento da abordagem (processo ou 
resultado), da natureza metodológica adotada e os principais temas investigados por estas pesquisas ao longo das últimas quatro décadas de pesquisa. Percebeu-se que tanto as pesquisas que a abordaram o consenso estratégico por resultado quando por processo, tiveram o enfoque dos seus estudos relacionados à influência. Quando o consenso foi abordado como processo, a preocupação latente foi em identificar como é a influência do consenso estratégico na prática estratégica. Já quando o tema foi abordado como resultado o interesse consistia em compreender o que é capaz de exercer influência no consenso estratégico. Curiosas e sutis diferenças que ficam ainda mais interessante quando relacionadas à metodologia adotada para compreensão desta influência. No primeiro caso, ocorre a predominância estudos quantitativos e no segundo qualitativos.

Baseado no que foi abordado, percebe-se que o consenso estratégico sob a ótica do seu estado da arte deixa lacunas a serem pesquisadas. Ainda é preciso consolidar o conceito do que é consenso estratégico. Pois atualmente o consenso estratégico é visto por múltiplas perspectivas, como por um meio de tomada de decisões, como um objetivo a ser alcançado ou como resultante de um processo conduzido de forma adequada. Sabe-se que o consenso no TMT contribui para a boa performance da organização, mas é replicável e é positivo nos demais níveis da organização? Sobre o que se deve buscar o consenso? Sempre é importante ter o consenso?

Em termos de tendências, pode-se indicar a tendência do incremente de estudos quantitativos, uma vez que as agendas de estudos futuros indicadas por este conjunto de 40 artigos analisados indicam a necessidade de validação dos modelos apresentados e a generalização dos resultados obtidos. Estudos estes que requerem a adoção de técnicas sofisticadas de pesquisa para que se possa de fato confirmar a existência de um modelo e a generalização de resultados. Outra indicação de estudos futuros recorrente, foi a observação de outras variáveis que possam exercer o papel de influenciadoras, tanto sob a ótica do que influencia o consenso estratégico quanto no que ele influência.

Explorando um pouco mais este ponto da influência do consenso estratégico é que fica o questionamento maior dos autores do presente estudo, que consiste em: o consenso estratégico sempre terá influência positiva na prática estratégia? Caso contrário, em quais contextos é desejável não obter consenso estratégico? Esta reflexão foi motivada pelos estudos de Camelo-ordaz, Hernández-lara \& Vallecabrera (2005) e Camelo-ordaz, Fernández-alles \& Hernández-lara (2010), nos quais os autores relacionam o consenso estratégico com a performance de inovação. A intenção dos autores não foi de identificar esta relação negativa do consenso estratégico como positiva para a performance da inovação, porém foram os primeiros achados, embora não fortes. Assim, o entendimento que se tem após o estudo realizado é que o tema do consenso estratégico ainda é um campo com múltiplas perspectivas e com potencial de pesquisa.

\section{REFERÊNCIAS}

Ambrosini, V. \& Bowman, C. (2003). Managerial consensus and corporate strategy: Why do executives agree or disagree about corporate strategy? European Management Journal, 21(2), 213-221.

Baranchuk, N. \& Dybvig, P. H. (2009). Consensus in diverse corporate boards. Review of Financial Studies, 22(2), 715-747.

Baucus, D. A., Baucus, M. S. \&Human, S. E. (1996). Consensus in franchise organizations: a cooperative arrangement among entrepreneurs. Journal of Business Venturing, 11(5), 359-378.

Bouillon, M. L., Ferrier, G. D., Stuebs Jr, M. T., \& West, T. D. (2006). The economic benefit of goal congruence and implications for management control systems. Journal of Accounting and Public Policy, 25(3), 265-298.

Bourgeois, L. J. Performance and consensus (1980). Strategic Management Journal, 1(3), 227-248.

Boyer, K. K. \& McDermott, C. (1999) Strategic consensus in operations strategy. Journal of Operations Management, 17(3), 289-305.

Byrne, S. \& Shrestha, G. (2014). A compromising consensus? Legitimising local government in postconflict Nepal. International Development Planning Review, 36(4), 435-453.

Camelo-ordaz, C., Hernández-lara, A. B. \& Vallecabrera, R. (2005). The relationship between top management teams and innovative capacity in companies. Journal of Management Development, 24(8), 683-705.

Camelo-ordaz, C., Fernández-alles, M. \& Hernándezlara, A. B. (2010) Strategic consensus, top management teams, and innovation performance. International Journal of Manpower, 31(6): 678695.

Dess, G. G. (1987). Consensus on strategy formulation and organizational performance: Competitors in a fragmented industry. Strategic management journal, 8(3), 259-277. 
Dess, G. G. \& Origer, N. K. (1987). Environment, structure, and consensus in strategy formulation: A conceptual integration. Academy of Management Review,12(2), 313-330.

De Woot, P., Heyvaert, H. \& Martou, F. (1977). Strategic management: an empirical study of 168 Belgian firms. International Studies of Management \& Organization, 60-75.

Ensley, M. D. \& Pearson, A. W. (2005). An exploratory comparison of the behavioral dynamics of top management teams in family and nonfamily new ventures: Cohesion, conflict, potency, and consensus. Entrepreneurship Theory and Practice, 29(3), 267-284.

Farjaudon, A. L. \& Morales, J. (2013). In search of consensus: The role of accounting in the definition and reproduction of dominant interests. Critical Perspectives on Accounting, 24(2), 154-171.

Floyd, S. W. \& Wooldridge, B. (1992). Managing strategic consensus: the foundation of effective implementation. The Executive, 6(4), 27-39.

Freling, T. H. \& Dacin, P. A. (2010). When consensus counts: Exploring the impact of consensus claims in advertising. Journal of Consumer Psychology, 20(2), 163-175.

Gorman, G. G., Rosa, P. J. \& Faseruk, A. (2005). Institutional lending to knowledge-based businesses. Journal of Business Venturing, 20(6), 793-819.

Grinyer, P. H. \& Norburn, D. (1977) Planning for existing markets: An empirical study. International Studies of Management \& Organization, 99-122.

Haddad, S. (2000) O estado da arte das pesquisas em Educação de Jovens e Adultos no Brasil: a produção discente da pós-graduação em educação no período 1986 - 1998. São Paulo: Ação Educativa.

Harris, M., Dopson, S. \& Fitzpatrick, R. (2009). Strategic drift in international non-governmental development organizations - putting strategy in the background of organizational change. Public Administration and Development, 29(5), 415-428.

Ho, J. Ly, Wu, A. \& Wu, S. Yc. (2014). Performance measures, consensus on strategy implementation, and performance: Evidence from the operationallevel of organizations. Accounting, Organizations and Society, 39(1), 38-58.
Holder Jr, J. J. (1976). Decision making by consensus. Readings in organizations: Behavior, structure, processes, 307-319.

Jaspers, F., Prencipe, A. \& Ende, J. (2012). Organizing interindustry architectural innovations: evidence from mobile communication applications. Journal of Product Innovation Management, 29(3), 419. 431.

Joshi, M. P., Kathuria, R. \& Porth, S. J. (2003). Alignment of strategic priorities and performance: an integration of operations and strategic management perspectives. Journal of Operations Management, 21(3), 353-369.

Kathuria, R., Porth, S. J., Kathuria, N. N., \& Kohli, T. K. (2010). Competitive priorities and strategic consensus in emerging economies: evidence from India. International Journal of Operations \& Production Management, 30(8), 879-896.

Kellermanns, F. W., Walter, J., Floyd, S. W., Lechner, C., \& Shaw, J. C. (2011). To agree or not to agree? A meta-analytical review of strategic consensus and organizational performance. Journal of Business Research, 64(2), 126-133.

Knight, D., Pearce, C. L., Smith, K. G., Olian, J. D., Sims, H. P., Smith, K. A., \& Flood, P. (1999). Top management team diversity, group process, and strategic consensus. Strategic Management Journal, 20(5), 445-465.

Khorshid, S. (2010). Soft consensus model based on coincidence between positive and negative ideal degrees of agreement under a group decisionmaking fuzzy environment. Expert Systems with Applications, 37(5), 3977-3985.

Lawrence, P. R.; Lorsch, J. (1967). Organization and Environment. Boston: Harvard University Press, $65,323-364$.

Marino, K. E. (1996). Developing consensus on firm competencies and capabilities. The Academy of Management Executive, 10(3), 40-51.

Markóczy, L. (2001). Consensus formation during strategic change. Strategic Management Journal, 22(11), 1013-1031.

McMahon, J. T. \& Ivancevich, J. M. (1976). A study of control in a manufacturing organization: Managers and nonmanagers. Administrative Science Quarterly, 66-83.

Murphy, T. \& O'brien, W. (2014). A strategic decision model for evaluating college and university 
sustainability investments. Management Research Review, 37(1), 2-18.

Noorderhaven, N. G., Benders, J. \& Keizer, Arjan B. (2007). Comprehensiveness versus pragmatism: consensus at the Japanese-Dutch interface. Journal of Management Studies, 44(8), 1349-1370.

Paraskevas, A. \& Saunders, M. NK. (2012). Beyond consensus: an alternative use of Delphi enquiry in hospitality research. International Journal of Contemporary Hospitality Management, 24(6), 907-924.

Phelps, J. E., Harris, T. E. \& Johnson, E. (1996). Exploring decision-making approaches and responsibility for developing marketing communications strategy. Journal of Business Research, 37(3), 217-223.

Pretorius, M. \& Maritz, R. (2011). Strategy making: the approach matters. Journal of Business Strategy, 32(4), 25-31.

Prusty, S. K., Mohapatra, P. K. J. \& Mukherjee, C. K. (2010). GOS tree (Goal-Objective-Strategy tree) approach to strategic planning using a fuzzy-Delphi process: An application to the Indian Shrimp Industry. Technological Forecasting and Social Change, 77(3), 442-456.

Quinn, J. B. (1982). Managing strategies incrementally. Omega, 10(6), 613-627.

Rapert, M. I., Velliquette, A. \& Garretson, J. A. (2002). The strategic implementation process: evoking strategic consensus through communication. Journal of Business Research, 55(4), 301-310.

Rasoolimanesh, S. M., Jaafar, M. \& Badarulzaman, N. (2014). Examining the contributing factors for the successful implementation of city development strategy in Qazvin City, Iran. Cities, 41, 10-19.

Stagner, R. (1969). Corporate decision making: An empirical study. Journal of Applied Psychology, 53(1), 1 .

Stoltzfus, K., Stohl, C. \& Seibold, D. R. (2011). Managing organizational change: paradoxical problems, solutions, and consequences. Journal of organizational change management, 24(3), 349367.

Tjosvold, D. \& Field, R. H. G. (1983). Effects of social context on consensus and majority vote decision making. Academy of Management Journal, 26(3), 500-506.

Vandenbosch, B. \& Saatcioglu, A. (2006). How managers generate ideas and why it matters. Journal of Business Strategy, 27(6): 11-17.

Young, S., Bartram, T., Staton, P. \& Leggat, S. G. (2010). High performance work systems and employee well-being: a two stage study of a rural Australian hospital. Journal of health organization and management, 24(2), 182-199.

Wooldridge, B. \& Floyd, S. W. (1989). Research notes and communications strategic process effects on consensus. Strategic Management Journal, 10(3), 295-302. 